\title{
Activation of IL-8 and its participation in cancer in schizophrenia patients: new evidence for the autoimmune hypothesis of schizophrenia
}

This article was published in the following Dove Medical Press journal: Neuropsychiatric Disease and Treatment

Lvzi Xu'

Xiao $\mathrm{Qi}^{2}$

Chi Zhu ${ }^{3}$

Lihua Wan'

'Department of Forensic Medicine, Chongqing Medical University, Chongqing, China; ${ }^{2}$ Department of Rehabilitation, Xinqiao Hospital, Third Military Medical University, Chongqing, China; ${ }^{3}$ Department of Neurology, Center for Clinical Neuroscience, Daping Hospital, Third Military Medical University, Chongqing, China
Correspondence: Lihua Wan Department of Forensic Medicine, Chongqing Medical University, \#I Yixueyuan Road, Yuzhong District, Chongqing 400000, China

Tel +862368485994

Fax +86 2368485994

Email ccfw@cqmu.edu.cn

\begin{abstract}
To investigate the autoimmune mechanisms of schizophrenia, we explored the relationship between schizophrenia and cancer using gene expression data of peripheral blood mononuclear cells from GSE27383 datasets. Gene screening and enrichment analysis using Gene Set Enrichment Analysis were applied to identify possible connections between schizophrenia and cancer. Real-time PCR (quantitative PCR), Western blotting and immunohistochemistry were performed on the brain tissue from both schizophrenia patients and normal controls. The genes for $I L-8$, as well as PTGS2, TPR, JUN, CXCL1, CXCL3, CXCL5 and PARD3 were highly expressed in schizophrenia patients. Cancer and chemokine signaling pathways were enriched in the schizophrenic group, related to the high expression of $I L-8$. Increased expression of IL-8 was further confirmed by quantitative PCR, Western blotting and immunohistochemistry results. Our results suggest that $I L-8$ may participate specifically in the pathophysiological changes that occur in schizophrenia. Additionally, our findings provide novel evidence supporting the autoimmune hypothesis of schizophrenia.
\end{abstract}

Keywords: bioinformatics, multiple primary cancers, autoimmune disease, cancer pathway, GSEA

\section{Introduction}

Schizophrenia is a severe, disabling neuropsychiatric disorder that affects $\sim 1 \%$ of the world's population. ${ }^{1,2}$ Although the exact causes of schizophrenia remain unknown, various etiological theories have been proposed to explain the pathogenesis of schizophrenia, including those which focus on developmental and neurodegenerative processes, genetic and environmental hypotheses or neurotransmitter abnormalities. ${ }^{2-6}$ In addition to these theories, the autoimmune hypothesis has been connected to several hypotheses explaining the etiopathogenesis of schizophrenia. ${ }^{7,8}$ Although cytokine expression in the brain tissue has been investigated considerably less often because of the limited numbers of brain samples obtained in previous studies, reports of immune activation in schizophrenic patients are generally in good consensus. There is increasing evidence from genomic, blood and in vivo imaging studies which reveal the involvement of immune activation in schizophrenia. Previous studies have reported that genetic regions containing cytokine IL-1a, IL-1b and IL-1RA are significantly associated with the human leukocyte antigen locus in schizophrenia., ${ }^{2,69}$ Serum cytokines including IL-6 and IL-8 are also significantly increased in schizophrenia patients. ${ }^{10-13}$ Elevated expression of immune-related genes other than cytokines has been found in the dorsolateral prefrontal cortex of individuals with schizophrenia. ${ }^{14-16}$ Brain microglia, which can synthesize and secrete cytokines along with resident immune cells, were found to 
be significantly activated using in vivo positron emission tomography in individuals with schizophrenia. ${ }^{17-19}$

Furthermore, subsequent studies have reported the similarities between schizophrenia and some autoimmune diseases. Epidemiological studies have shown an increased risk of developing several autoimmune diseases such as type I diabetes mellitus and thyrotoxicosis. ${ }^{20}$ One study reported that the prevalence of antithyroid antibodies was almost doubled in both male and female schizophrenia patients. ${ }^{21}$ Levels of autoantibodies against the brain or specific areas of the brain (cerebrum, frontal cortex and septal areas) were found to increase in people with schizophrenia. ${ }^{22-24}$ Thus, our improved understanding of the immune system has provided more evidence for the autoimmune theory of schizophrenia. Another study showed that autoantibodies extracted and purified from the blood of schizophrenia patients specifically target muscarinic acetylcholine receptor, a neurotransmitter receptor. This indicates that functional autoantibodies are capable of modulating neurotransmission and may play an important role in the pathogenesis of schizophrenia. ${ }^{25}$ However, to verify the autoimmune hypothesis, more evidence needs to be provided by future investigations.

In recent years, there have been many studies reporting results based on genetic research into schizophrenia, including the discovery of schizophrenia candidate genes and their corresponding pathways. Based on further analysis and verification of known and updated pathways, several pathway databases are currently available for analyzing functional interactions between genes and pathways in schizophrenia. These include the Database for Annotation, Visualization and Integrated Discovery; the Kyoto Encyclopedia of Genes and Genomes; Reactome, Database of Interacting Proteins and ConsensusPathDB. ${ }^{26-30}$ However, in the field of genetic association research, results reported to date have tended to be inconsistent. ${ }^{31}$ Compared to focusing on a single gene or pathway, functional evaluation of entire databases should reveal more information about the pathogenesis of schizophrenia. In this study, we aimed to investigate the autoimmune basis of schizophrenia by conducting a systematic genetic association analysis on data from schizophrenia patients using bioinformatic methods.

\section{Materials and methods}

\section{Bioinformatic analysis}

\section{Data sources}

Microarray data were obtained from the Gene Expression Omnibus database on the National Center for Biotechnology Information website (http://www.ncbi.nlm.nih.gov/geo).
We chose dataset GSE27383 and six cases each from the normal people and schizophrenia patient samples. ${ }^{32}$ Dataset GSE27383 contains whole-genome peripheral blood mononuclear cell gene expression data from males for both recent-onset schizophrenia patients $(<5$ years $)$ and normal controls. More details can be found at https://www.ncbi.nlm. nih.gov/geo/query/acc.cgi?acc=GSE27383.

\section{Data processing}

Gene Set Enrichment Analysis (GSEA; http://www. broadinstitute.org/gsea) was used to perform enrichment analysis. ${ }^{33}$ GSE27383 datasets were divided into two groups: schizophrenia patients and normal controls. Molecular Signatures Database (MSigDB) c5.bp.v5.1, c5.c c.v5.1 and c5.mf.v5.1 were selected as reference gene sets, including a total of 1,454 biological processes, cellular components and molecular functions. Enrichment of the related gene set was analyzed according to the default statistical methods for weighted enrichment, with the random repeat number set to 1,000 , false discovery rate (FDR) $<25 \%$ and the standardized $P<0.05$ defined as positive. ${ }^{34}$

\section{Experiments using brain tissues}

\section{Brain samples}

Samples of prefrontal cortex were collected from six schizophrenia patients and six controls during autopsy at the Chongqing Engineering Center for Criminal Investigation Technology (Table 1). The six cases of schizophrenia were confirmed by a psychiatrist before their death. All the deceased died of either suicide, homicide or an accident. Both the schizophrenia patients and the controls were confirmed as not having cancer by an anatomical and pathological

Table I General information about the deceased patients in this study

\begin{tabular}{l|l|l|l}
\hline Groups & Sex & Age, years & $\begin{array}{l}\text { Cause of } \\
\text { death }\end{array}$ \\
\hline Schizophrenia & Male & 46 & Accident \\
patients & Male & 32 & Accident \\
& Male & 45 & Accident \\
& Male & 57 & Suicide \\
& Female & 43 & Accident \\
Normal controls & Female & 55 & Suicide \\
& Male & 35 & Accident \\
& Male & 43 & Accident \\
& Male & 41 & Homicide \\
& Male & 55 & Accident \\
& Male & 44 & Accident \\
& Male & 59 & Accident \\
\hline
\end{tabular}

Note: This table shows the age, sex and cause of death of the deceased patients from whom brain samples were obtained. 
examination. This study was approved by the Chongqing Medical University ethics committee.

\section{Real-time PCR}

RNA was extracted from the brain tissues of schizophrenia patients and normal controls using a mirVana miRNA Isolation Kit (Ambion, Austin, TX, USA). A PrimeScript RT Reagent Kit (Takara, Shiga, Japan) was used to perform reverse transcription, followed by quantitative PCR (qPCR) with SYBR Premix Ex Taq II (Takara), using the Step One qPCR system program. Each gene group was assigned three wells, with reduced glyceraldehyde-phosphate dehydrogenase (GAPDH) as the control. PCR primer sequences were selected from the PrimerBank (https://pga. mgh.harvard.edu/primerbank/). The primer sequences were as follows: GAPDH (GCACCGTCAAGGCTGAGAAC, TGGTGAAGACGCCAGTGGA) and IL-8 (ATGACTTC CAAGCTGGCCGTG, GGCCAGCCAGGTCCAGAC).

\section{Immunohistochemistry}

Brain tissues of schizophrenia patients and normal controls were first embedded in paraffin, cut into $5 \mu \mathrm{m}$ sections and heated at $70^{\circ} \mathrm{C}$ in the oven for 6 hours. Sections were dewaxed in xylene and brought to water through graded alcohols. IL-8 antibody (1:500; Abcam, Cambridge, UK) and secondary antibody (1:5,000; Abcam) were then added. Sections were rinsed three times with PBS, incubated with 3,3'-diaminobenzidine, stained with hematoxylin and finally fixed with neutral balata.

\section{Western blotting}

Total protein from prepared tissues was extracted using RIPA lysis buffer, and the protein concentration was determined using the Bradford assay according to the manufacturer's instructions. A balanced protein was electrophoresed by SDS-PAGE at $150 \mathrm{~V}$ for 100 minutes. Then, the protein was transferred to a polyvinylidene fluoride (PVDF) membrane according to the wet transfer method (400 mA, 60 minutes). The PVDF membrane was blocked using evaporated milk and incubated overnight at $4^{\circ} \mathrm{C}$ with the primary antibody $(1: 1,000)$. The PVDF membrane was then incubated for 1 hour at room temperature with the secondary antibody $(1: 10,000)$. Lastly, the protein band was developed on the film using a chemiluminescence kit (Sangon Biotech, Shanghai, China).

\section{Statistical analysis}

Expression levels of IL-8 in schizophrenia patients and normal controls from the GSE27383 dataset were compared with $t$-tests using SPSS 17.0 (SPSS Inc., Chicago, IL, USA). A significance level of $P<0.05$ was defined as being statistically significant.

\section{Results \\ Bioinformatic analysis}

Microarray dataset GSE27383 was extracted from the Gene Expression Omnibus database, and data from ten schizophrenia patients and ten normal controls were analyzed using GSEA software. Results showed that the $I L-8$ expression level was significantly higher in schizophrenia patients than controls ( $P=0.002$; Figure 1B). A heat map of the top 50 differentially expressed genes is shown in Figure 1A. The results from GSEA were ranked according to the normalized enrichment score (NES) and their order suggested that cancer- and chemokine signaling pathway-related genes were enriched in schizophrenia patients with high expression of $I L-8$ (Figure 2). On the heat maps (Figures $1 \mathrm{~A}$ and 2), the expression values are represented as colors, where the range of colors (red, pink, light blue, dark blue) shows the range of expression levels (high, moderate, low, lowest). The enrichment score (ES) reflects the degree to which a gene set is overrepresented at the top or bottom of a ranked list of genes. GSEA calculates the ES by walking down the ranked list of genes, increasing a running-sum statistic when a gene is in the gene set and decreasing it when it is not. The magnitude of the increment depends on the correlation of the gene with the phenotype. The ES is the maximum deviation from zero encountered in walking the list. A positive ES indicates gene set enrichment at the top of the ranked list and a negative ES indicates gene set enrichment at the bottom of the ranked list. The NES is the primary statistic for examining gene set enrichment results. By normalizing the ES, GSEA accounts for differences in gene set size and in correlations between gene sets and the expression dataset; therefore, the NES can be used to compare analysis results across gene sets. The nominal $P$-value estimates the statistical significance of the ES for a single gene set. ${ }^{35}$

Tables 2 and 3 show the identified genes from the cancer and chemokine signaling pathways. There were 13 coreenriched genes from the cancer pathway and 7 core-enriched genes from the chemokine signaling pathway. $I L-8$ appeared in both pathways, meaning that $I L-8$ is at the intersection of the cancer and chemokine signaling pathways. The top three genes from the cancer pathway and the top four genes from the chemokine signaling pathway, excluding $I L-8$ in both cases, were selected to analyze correlations with $I L-8$ expression (Figure 3 ). The correlation analysis indicated a 
A

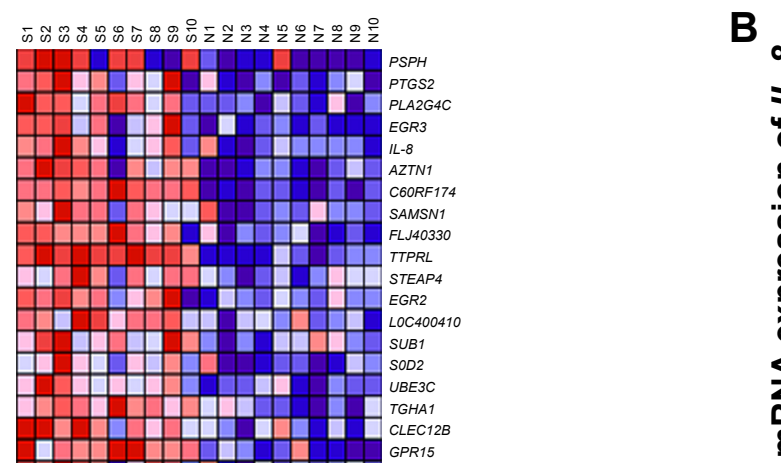

B

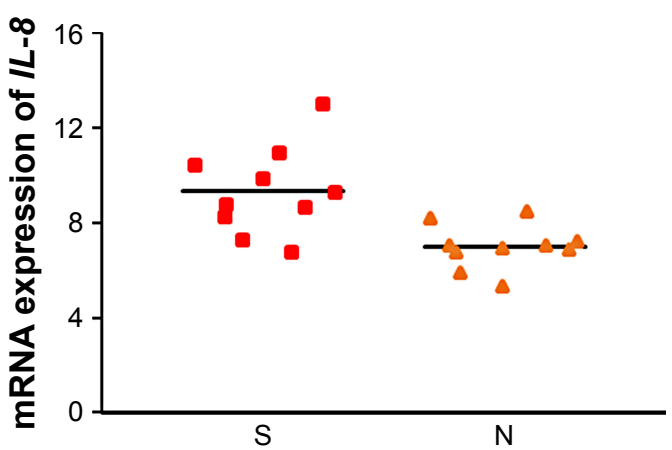

Figure I Results of GSEA analysis. SI-SIO refers to the ten samples from schizophrenia patients and $\mathrm{NI}-\mathrm{NIO}$ refers to the ten samples from controls.

Notes: (A) Data from ten schizophrenia patients and ten normal controls were extracted from the microarray dataset GSE27383 and analyzed using GSEA. Gene expression differences between the two groups are shown as heat maps of the top 50 DEGs. (B) mRNA expression of IL-8 in schizophrenia patients (S) and normal controls (N). Expression of IL-8 mRNA in peripheral blood of schizophrenia patients is significantly increased.

Abbreviations: DEGs, differentially expressed genes; GSEA, Gene Set Enrichment Analysis.

significant positive correlation between $I L-8$ and PTGS2, $J U N$ and $C X C L 1$. The rank in the gene list of these genes was $<30$. $P$-values of correlations between the cancer and chemokine signaling pathways were adjusted using the Bonferroni correction to control for the family-wise error rate (Table 4). Although the FWER $P$-value was $>0.05$, the goal of GSEA is to generate hypotheses, so the GSEA team recommends focusing on the FDR statistic. ${ }^{35}$

\section{IL-8 expression in brain tissue}

Analysis with qPCR showed that IL-8 expression was significantly increased in the brain tissue of schizophrenia patients compared to controls (Figure 4A and B). Results from Western blotting showed the same trend. Furthermore, the increased expression of IL-8 was confirmed in the immunohistochemistry results (Figure 4C).

\section{Discussion}

Schizophrenia is a severe, disabling neuropsychiatric disorder whose exact cause remains unknown. With better understanding of the pathogenesis of schizophrenia and increasing research into the immune system, the autoimmune hypothesis has been receiving increasing attention. Despite numerous reports describing immune or autoimmune abnormalities in schizophrenia, the hypothesis that these abnormalities are related to the pathogenesis of the disease is still in question. Thus, we conducted a systematic genetic association analysis of schizophrenia patients to test the autoimmune hypothesis. This analysis identified the genes involved in inflammation 


\section{A}
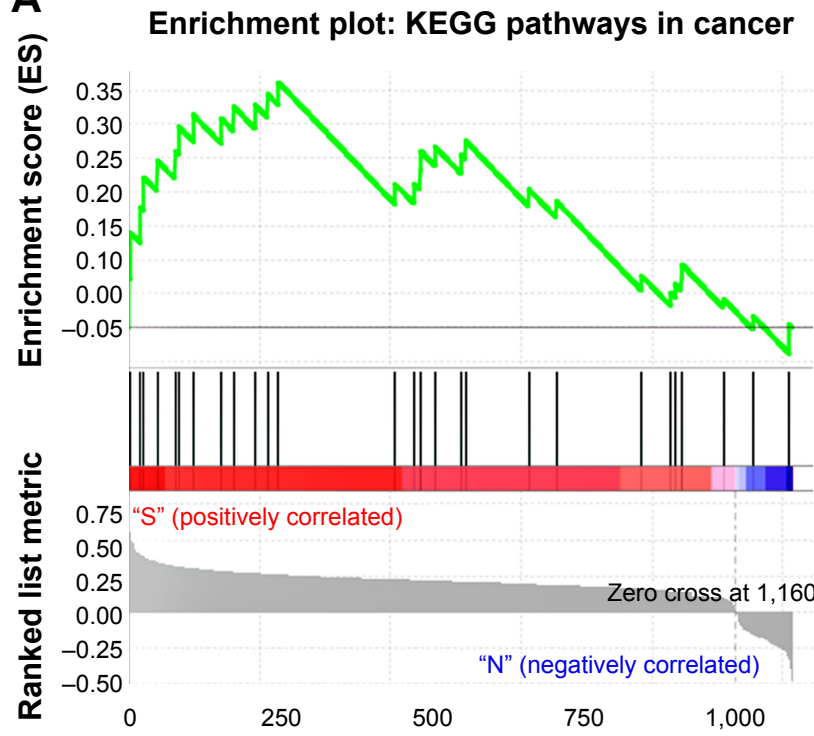

Rank in ordered dataset

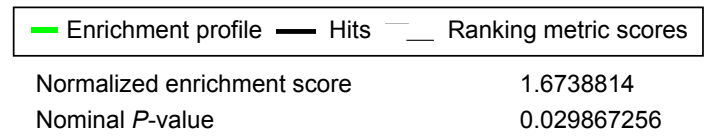

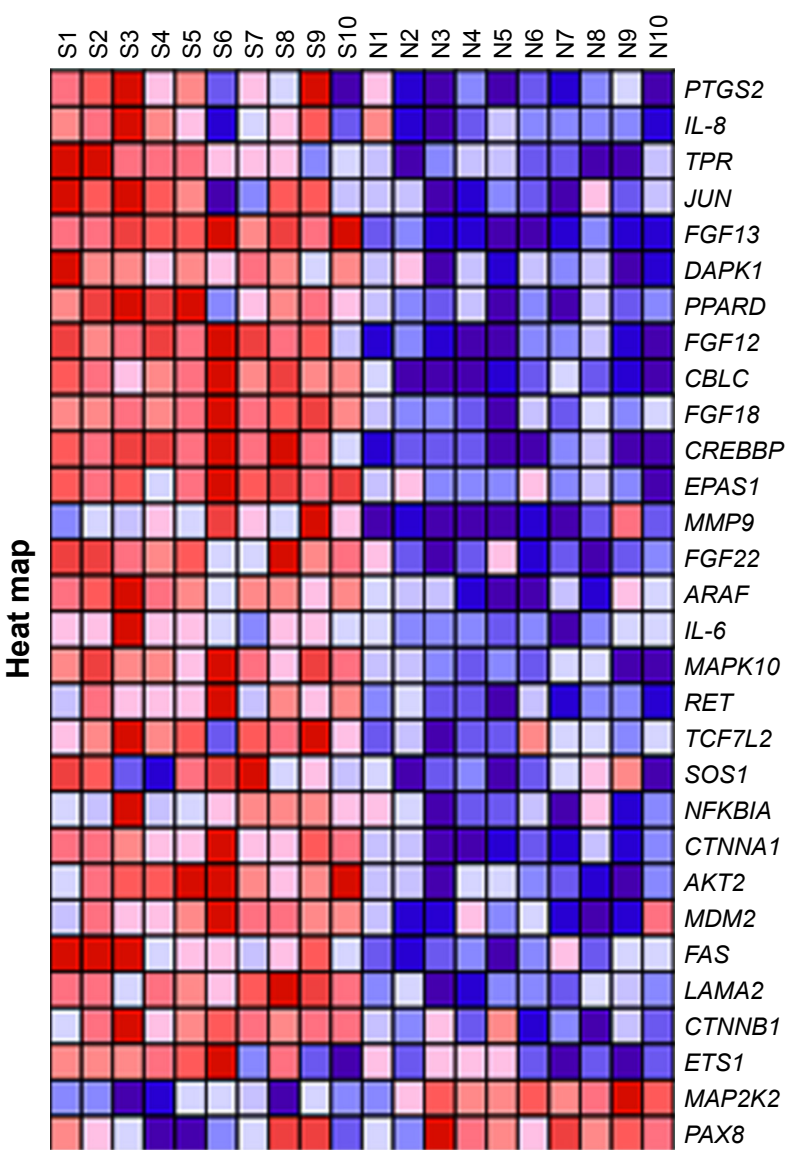

B
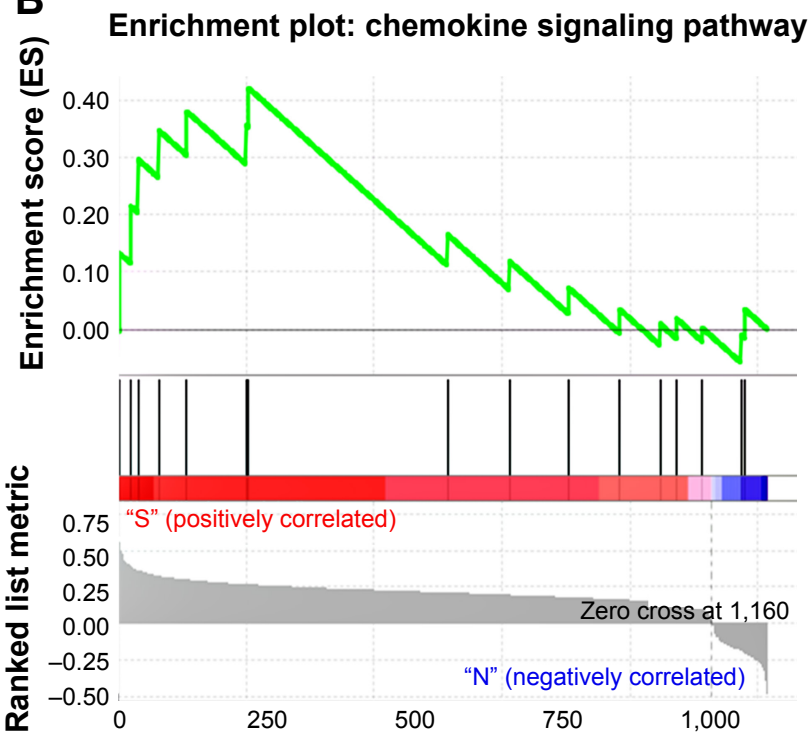

Rank in ordered dataset

\begin{tabular}{|c|c|}
\hline - Enrichment profile — Hits & __ Ranking metric scores \\
\hline Normalized enrichment score & 1.615543 \\
\hline Nominal $P$-value & 0.042875156 \\
\hline
\end{tabular}

Figure 2 Results of enrichment analysis.

Notes: Gene expression differences between schizophrenia patients and normal controls were ranked according to the NES. In schizophrenia patients, both the cancer pathway $(\mathbf{A})$ and the chemokine signaling pathway $(\mathbf{B})$ were enriched around the increased expression of IL-8 $(P<0.05)$.

Abbreviations: KEGG, Kyoto Encyclopedia of Genes and Genomes; NES, normalized enrichment score. 
Table 2 Members of the gene set in the cancer pathway

\begin{tabular}{|c|c|c|c|c|c|}
\hline Gene symbol & $\begin{array}{l}\text { Gene location on } \\
\text { chromosome }\end{array}$ & Rank in gene list & Rank metric score & Running ES & Core enrichment \\
\hline PTGS2 & Iq31.I & I & 0.563 & 0.0726 & Yes \\
\hline IL-8 & $4 q 13.3$ & 4 & 0.528 & 0.1399 & Yes \\
\hline TPR & $\mid q 31.1$ & 23 & 0.390 & 0.1763 & Yes \\
\hline JUN & Ip32.| & 29 & 0.378 & 0.2216 & Yes \\
\hline FGF/3 & Xq26.3-q27.I & 55 & 0.343 & 0.2462 & Yes \\
\hline DAPKI & $9 q 21.33$ & 89 & 0.314 & 0.2606 & Yes \\
\hline PPARD & $6 p 21.31$ & 96 & 0.311 & 0.2963 & Yes \\
\hline$F G F / 2$ & $3 q 28-q 29$ & 124 & 0.299 & 0.3136 & Yes \\
\hline CBLC & $19 q 13.32$ & 177 & 0.282 & 0.3084 & Yes \\
\hline FGFI8 & $5 q 35.1$ & 201 & 0.275 & 0.3257 & Yes \\
\hline CREBBP & $|6 p| 3.3$ & 242 & 0.266 & 0.3281 & Yes \\
\hline EPASI & $2 p 21$ & 266 & 0.261 & 0.3437 & Yes \\
\hline MMP9 & $20 q 13.12$ & 287 & 0.257 & 0.3610 & Yes \\
\hline FGF22 & $19 p \mid 3.3$ & 510 & 0.224 & 0.2111 & No \\
\hline ARAF & XpII.3 & 546 & 0.220 & 0.2115 & No \\
\hline IL-6 & $7 p \mid 5.3$ & 557 & 0.218 & 0.2319 & No \\
\hline MAPKIO & $4 q 21.3$ & 558 & 0.217 & 0.2603 & No \\
\hline RET & $10 q \mid 1.21$ & 586 & 0.214 & 0.2664 & No \\
\hline TCF7L2 & $10 q 25.2-q 25.3$ & 636 & 0.208 & $0.254 I$ & No \\
\hline SOSI & $2 p 22.1$ & 644 & 0.207 & 0.2754 & No \\
\hline NFKBIA & $|4 q| 3.2$ & 765 & 0.191 & 0.2035 & No \\
\hline CTNNAI & $5 q 31.2$ & 817 & 0.184 & 0.1864 & No \\
\hline AKT2 & $19 q 13.2$ & 981 & 0.160 & 0.0757 & No \\
\hline MDM2 & $12 q 15$ & 1,036 & 0.147 & 0.0513 & No \\
\hline FAS & $10 q 23.31$ & 1,045 & 0.145 & 0.0638 & No \\
\hline LAMA2 & $6 q 22.33$ & 1,056 & 0.143 & 0.0744 & No \\
\hline CTNNBI & $3 p 22.1$ & 1,057 & 0.143 & 0.0931 & No \\
\hline ETSI & I Iq24.3 & 1,137 & 0.097 & 0.0420 & No \\
\hline MAP2K2 & $|9 p| 3.3$ & 1,193 & -0.151 & 0.0173 & No \\
\hline PAX8 & $2 q 14.1$ & 1,263 & -0.325 & 0.0040 & No \\
\hline
\end{tabular}

Note: A "yes" in the last column (core enrichment) indicates core enrichment of the corresponding gene in the cancer pathway.

Abbreviation: ES, enrichment score.

Table 3 Members of the gene set in the chemokine signaling pathway

\begin{tabular}{|c|c|c|c|c|c|}
\hline Gene symbol & $\begin{array}{l}\text { Gene location on } \\
\text { chromosome }\end{array}$ & Rank in gene list & Rank metric score & Running ES & Core enrichment \\
\hline IL-8 & $4 q 13.3$ & 4 & 0.528 & 0.1324 & Yes \\
\hline CXCLI & $4 q 13.3$ & 26 & 0.384 & 0.2143 & Yes \\
\hline CXCL5 & $4 q 13.3$ & 40 & 0.360 & 0.2964 & Yes \\
\hline CXCL3 & $4 q \mid 3.3$ & 80 & 0.320 & 0.3475 & Yes \\
\hline PARD3 & $|0 p||.22-p||.2|$ & 135 & 0.296 & 0.3805 & Yes \\
\hline DOCK2 & $5 q 35.1$ & 250 & 0.264 & 0.3573 & Yes \\
\hline CCRIO & $|7 q 2| .2$ & 256 & 0.264 & 0.4211 & Yes \\
\hline SOSI & $2 p 22.1$ & 644 & 0.207 & 0.1653 & No \\
\hline NFKBIA & $|4 q| 3.2$ & 765 & 0.191 & 0.1186 & No \\
\hline VAV3 & $|p| 3.3$ & 880 & 0.175 & 0.0726 & No \\
\hline AKT2 & $19 q \mid 3.2$ & 981 & 0.160 & 0.0339 & No \\
\hline JAK3 & $19 p|3.1|$ & 1,059 & 0.142 & 0.0090 & No \\
\hline CCR9 & $3 p 21.31$ & 1,092 & 0.134 & 0.0179 & No \\
\hline CCR7 & $|7 q 2| .2$ & $\mathrm{I}, 142$ & 0.088 & 0.0014 & No \\
\hline$A D R B K 2$ & $22 q 12.1$ & 1,217 & -0.185 & -0.0101 & No \\
\hline CCL28 & $5 p / 2$ & $\mathrm{I}, 224$ & -0.195 & 0.0351 & No \\
\hline
\end{tabular}

Note: A "yes" in the last column (core enrichment) indicates core enrichment of the corresponding gene in the chemokine signaling pathway.

Abbreviation: ES, enrichment score. 

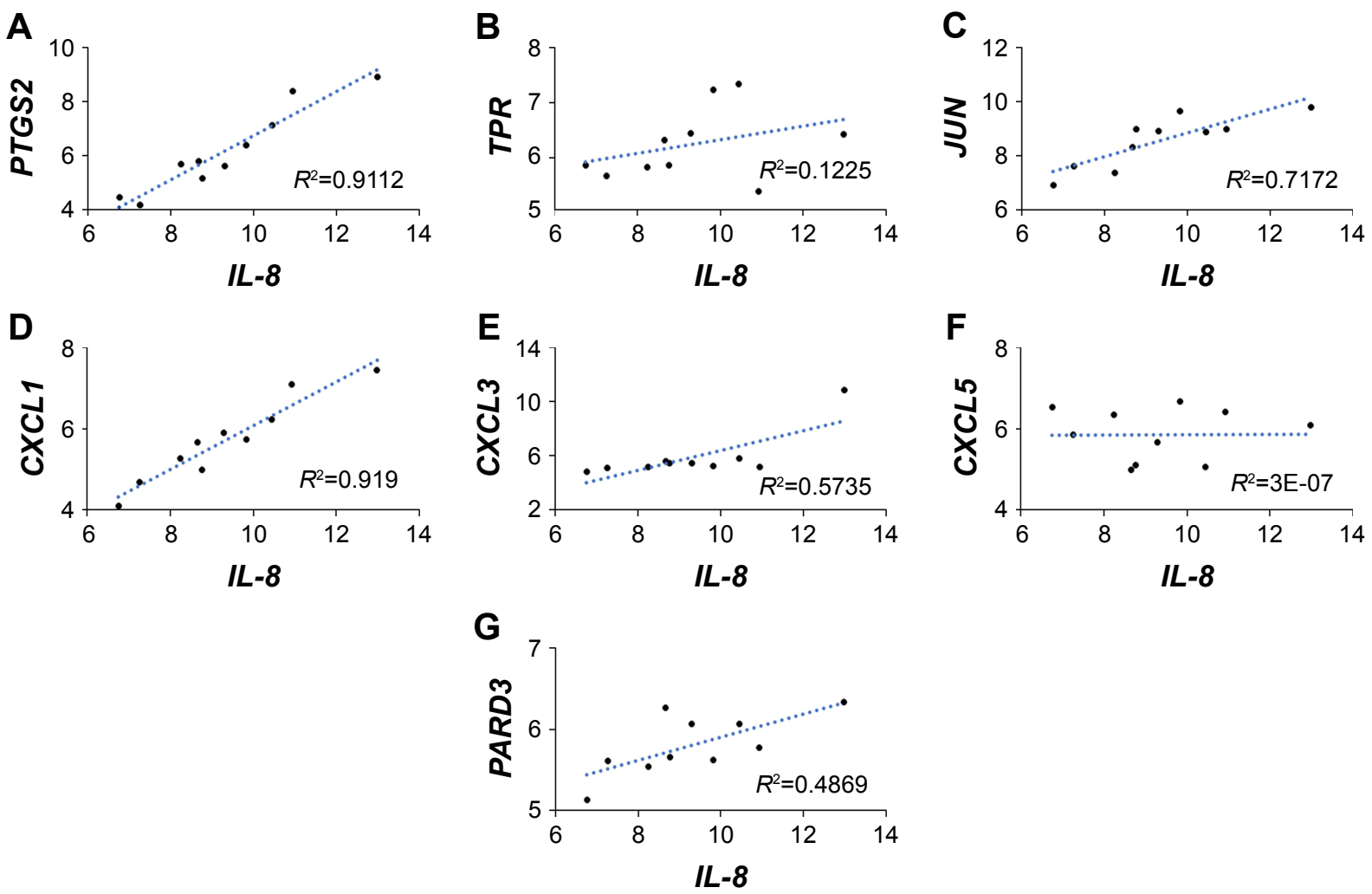

Figure 3 Cancer-related genes and IL-8.

Notes: (A-G) GSEA results showed that the expression levels of several cancer-related genes, including PTGS2, TPR, JUN, CXCLI, CXCL3, CXCL5 and PARD3, were significantly increased in schizophrenia patients and that the increased expression of each was correlated with the increased expression of IL-8.

Abbreviation: GSEA, Gene Set Enrichment Analysis.

and cancer, specifically genes coding for IL-8, PTGS2, TPR, JUN, CXCL1, CXCL3, CXCL5 and PARD3, all of which showed increased expression in schizophrenia patients. Furthermore, we also observed that cancer- and chemokine signaling pathway-related genes were enriched in schizophrenia patients who had high expression of IL-8. We further confirmed the increased expression of IL-8 by performing qPCR, Western blotting and immunohistochemistry. This implies a novel, important connection between schizophrenia and cancer that provides further evidence for the autoimmune hypothesis.

Autoimmune diseases and cancer are both induced by disruption of the balance between the capacity to recognize

Table $4 P$-values and FWER $P$-values for gene sets

\begin{tabular}{l|l|l}
\hline Pathway & P-value & FWER P-value \\
\hline Chemokine signaling pathway & 0.030 & 0.387 \\
Cancer pathway & 0.043 & 0.498 \\
\hline
\end{tabular}

Notes: $P$-values were adjusted using the Bonferroni correction to yield the FWER $P$-value. FWER $P$-values $>0.05$ indicate nonsignificant effects. According to the user guide of GSEA software, the software development team suggest the use of FDR and NES, instead of FWER, for evaluate the results of GSEA.

Abbreviations: FWER, family-wise error rate; GSEA, Gene Set Enrichment Analysis. and fight exogenous molecules and the capacity to avoid autoreactivity. ${ }^{36}$ Although autoimmunity in cancer is yet to be thoroughly investigated, increasing numbers of studies suggest that aggravated autoimmune responses play a crucial role in manifestation of cancer. Autoantibody targets such as IGHG4, CRYM, EFCAB2, STAT6, HDAC7A and CCNB1 were significantly deregulated across all grades of meningioma. ${ }^{37}$ In cancer, both active and passive immunotherapies have been tested with promising results, which is similar to the immunotherapies applied to several autoimmune diseases. ${ }^{38-41}$ Building on our understanding of autoimmunity in cancer, in this paper, we have provided further evidence of the autoimmune hypothesis of schizophrenia by revealing a novel connection between schizophrenia and cancer.

In this study, we analyzed microarray data from the GSE27383 dataset to screen for susceptibility genes in schizophrenia and found that the expression of IL-8 was increased in schizophrenia. Our results confirm the role of IL-8 in pathological processes underlying schizophrenia. Previous studies have mainly focused on the nonspecific 
A

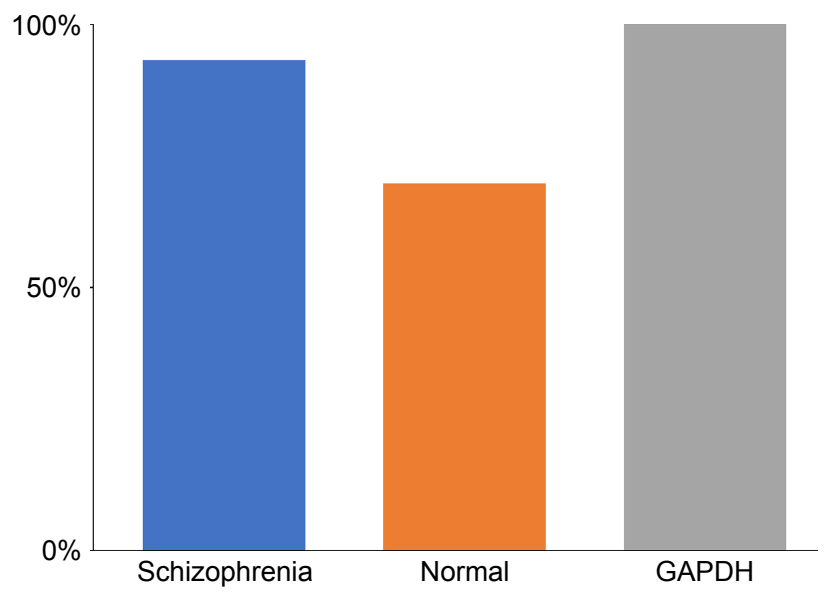

B

C
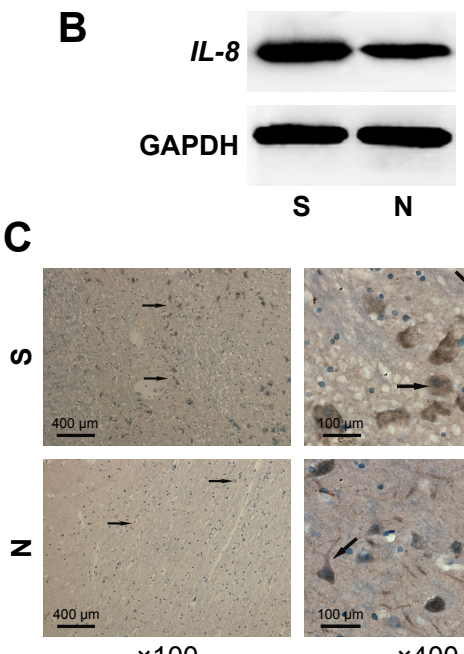

$\times 100$

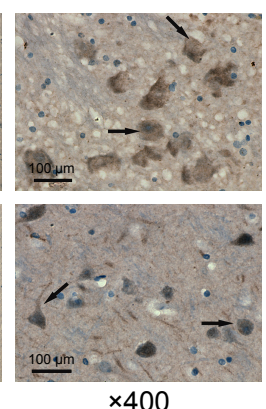

Figure 4 Increased expression of IL-8 among schizophrenia patients.

Notes: "S" means schizophrenia patients and "N" means normal control. IL-8 was found to be significantly increased in the prefrontal cortex of schizophrenia patients according to results from PCR; the $X$ axis represents different groups and the $Y$ axis represents the relative percentage of GAPDH (A), Western blotting (B), and immunohistochemistry $(\mathbf{C})$.

Abbreviation: GAPDH, glyceraldehyde-phosphate dehydrogenase.

association of chemokines, including IL-8, IL-6, interferon- $\gamma$ and macrophage inflammatory protein 1 gamma, with psychiatric disorders. ${ }^{42-44}$ However, growing evidence suggests that specific cytokines may play a role in many neurobiological processes that are potentially relevant to psychiatric disorders, beyond their classical chemotactic functions. In a recent study, upregulation of IL-8 in the anterior cingulate cortex was reported to be involved in prefrontal synaptic transmission. ${ }^{45}$ Moreover, immune reactivities mediated by IL-8 have important implications for autoimmune diseases. ${ }^{43}$ IL-8 may also participate in the pathogenesis of autoimmune diseases by mediating the formation of neutrophil extracellular traps. ${ }^{46}$ In agreement with these findings, our results suggest that IL-8 specifically participates in the pathophysiological changes accompanying schizophrenia, but further investigation is required to confirm this.

In addition, we found that the expression levels of several genes were increased in schizophrenia patients. Among all the candidate genes, $I L-8$ and several cancer-related genes, including PTGS2, TPR, JUN, CXCL1, CXCL3, CXCL5 and $P A R D 3$, were further analyzed to examine the correlations based on GSEA enrichment results. The correlation analysis indicated that a significant positive correlation existed between $I L-8$ and $P T G S 2$ and between $J U N$ and $C X C L 1$. $P T G S 2$ has been reported to be overexpressed in cancers and is thought to regulate cancer cell migration and invasion, since these processes are inhibited by knocking down COX2-overexpressing cancer cells. ${ }^{47,48}$ Likewise, $J U N$ has been reported to be related to cancer. ${ }^{49-51} C X C L 1$ was found to be critical to metastasis and cancer growth in many cancers. ${ }^{52}$ Moreover, cancer- and chemokine signaling pathway-related genes have been enriched in schizophrenia in this study, indicating a strong relationship between schizophrenia and cancer.

The connection between schizophrenia and cancer has also been reported in several previous studies. Epidemiological studies show that schizophrenia patients suffer a lower incidence of lung and bladder cancer and that the decreased risk of cancer is related to increased duration and age of onset of schizophrenia. ${ }^{53-55}$ Recent genetic research has also reported some correlations between schizophrenia and cancer, including a shared protein transcription pathway. ${ }^{56,57}$ Thus, different kinds of cancers might develop through the same protein pathway. This would explain why multiple primary cancers are not common. ${ }^{58-60}$ By comparing the hallmarks, emerging hallmarks and enabling characteristics of melanoma to schizophrenia, Brown et al proposed that schizophrenia is a condition of attenuated tumorigenesis. ${ }^{61}$ Assuming that the pathophysiology of schizophrenia can be considered as resembling that of a tumor due to the shared protein pathway, schizophrenia and cancer may compete at the level of these pathways as they develop in individual patients. Other studies have shown that the risk of a second primary cancer is increased after treatment of the first primary cancer. ${ }^{62-64}$ This may be because pathogenic factors have not been removed, whereas the pathways have been released after the first primary cancer was suppressed or resected. At present, the pathophysiological processes 
underlying schizophrenia are still unknown, so schizophrenia is treated using antipsychotics and psychological interventions. ${ }^{65}$ This may indicate that the pathogenic factors underlying schizophrenia are not removed by such treatments and that pathways shared between schizophrenia and cancer are occupied by schizophrenia. This would explain why schizophrenia patients have a low risk of cancer development. ${ }^{66,67}$

Although the results of our study are promising, we fully acknowledge its limitations. Firstly, due to the limited amount of brain tissue we were able to obtain for the study, we failed to fully confirm overexpression of IL-8 in the brain tissue from schizophrenia patients. Secondly, the specific autoimmune response involved in the pathological process underlying schizophrenia has not yet been investigated.

Further studies are warranted to find more specific autoantibodies from schizophrenia patients. With the discovery of specific antibody-mediated autoimmune responses, more effective treatments and novel strategies for immunotherapy will likely be created for schizophrenia patients in the future.

\section{Conclusion}

This study is one of the first comprehensive investigations of schizophrenia patients to explore the autoimmune mechanisms underlying schizophrenia and its relationship with cancer. Our results confirm overexpression of IL-8 in schizophrenia patients and reveal novel connections between schizophrenia and cancer. In addition, by providing a novel understanding of the autoimmunity of cancer, we further clarify the autoimmune basis of schizophrenia.

\section{Acknowledgments}

We thank Chongqing Engineering Center for Criminal Investigation Technology for providing brain samples. We acknowledge Dr Guang Li for his assistance during the experiments and for valuable discussions.

\section{Disclosure}

The authors report no conflicts of interest in this work.

\section{References}

1. Freedman R. Schizophrenia. N Engl J Med. 2003;349(18):1738-1749.

2. Ganesh S, Ashok AH, Kumar CN, Thirthalli J. Prevalence and determinants of metabolic syndrome in patients with schizophrenia: a systematic review and meta-analysis of Indian studies. Asian J Psychiatr. 2016; 22:86-92.

3. Hornig M, Mervis R, Hoffman K, Lipkin WI. Infectious and immune factors in neurodevelopmental damage. Mol Psychiatry. 2002;7(2): S34-S35.
4. Rund BR. Is schizophrenia a neurodegenerative disorder? Nord $J$ Psychiatry. 2009;63(3):196-201.

5. Aghajanian GK, Marek GJ. Serotonin model of schizophrenia: emerging role of glutamate mechanisms. Brain Res Rev. 2000;31(2-3): 302-312.

6. Harrison PJ, Weinberger DR. Schizophrenia genes, gene expression, and neuropathology: on the matter of their convergence. Mol Psychiatry. 2005;10(1):40-68.

7. Na KS, Jung HY, Kim YK. The role of pro-inflammatory cytokines in the neuroinflammation and neurogenesis of schizophrenia. Prog Neuropsychopharmacol Biol Psychiatry. 2014;48:277-286.

8. Jones AL, Mowry BJ, Pender MP, Greer JM. Immune dysregulation and self-reactivity in schizophrenia: do some cases of schizophrenia have an autoimmune basis? Immunol Cell Biol. 2005;83(1):9-17.

9. $\mathrm{Xu} \mathrm{M}, \mathrm{He} \mathrm{L}$. Convergent evidence shows a positive association of interleukin-1 gene complex locus with susceptibility to schizophrenia in the Caucasian population. Schizophr Res. 2010;120(1-3):131-142.

10. Kunz M, Ceresér KM, Goi PD, et al. Serum levels of IL-6, IL-10 and TNF- $\alpha$ in patients with bipolar disorder and schizophrenia: differences in pro- and anti-inflammatory balance. BrazJPsychiatr. 2011;33(3):268-274.

11. Lin A, Kenis G, Bignotti S, et al. The inflammatory response system in treatment-resistant schizophrenia: increased serum interleukin-6. Schizophr Res. 1998;32(1):9-15.

12. Zhang XY, Zhou DF, Cao LY, Zhang PY, Wu GY, Shen YC. Changes in serum interleukin-2, -6 , and -8 levels before and during treatment with risperidone and haloperidol: relationship to outcome in schizophrenia. J Clin Psychiatry. 2004;65(7):940-947.

13. Avguštin $B$, Wraber $B$, Pesek MB, Tavcar R. Increased Th1 and Th2 immune reactivity with relative Th2 dominance in patients with acute exacerbation of schizophrenia and chronic schizophrenia. Brain Behav Immun. 2005;19(4):268-274.

14. Doorduin J, de Vries EF, Willemsen AT, de Groot JC, Dierckx RA, Klein HC. Neuroinflammation in schizophrenia-related psychosis: a PET study. J Nucl Med. 2009;50(11):1801-1807.

15. van Berckel BN, Bossong MG, Boellaard R, et al. Microglia activation in recent-onset schizophrenia: a quantitative (R)-11PK11195 positron emission tomography study. Biol Psychiatry. 2008;64(9):820-822.

16. Kurumaji A, Wakai T, Toru M. Decreases in peripheral-type benzodiazepine receptors in postmortem brains of chronic schizophrenics. J Neural Transm (Vienna). 1997;104(11-12):1361-1370.

17. Wolf SA, Boddeke HW, Kettenmann H. Microglia in physiology and disease. Anпu Rev Physiol. 2017;79(1):619-643.

18. Frank MG, Baratta MV, Sprunger DB, Watkins LR, Maier SF. Microglia serve as a neuroimmune substrate for stress-induced potentiation of CNS pro-inflammatory cytokine responses. Brain Behav Immun. 2007; 21(1):47-59.

19. Aloisi F. Immune function of microglia. Glia. 2001;36(2):165-179.

20. Wright P, Sham PC, Gilvarry CM, Jones PB, Cannon M, Sharma T, Murray RM. Autoimmune diseases in the pedigrees of schizophrenic and control subjects. Schizophr Res. 1996;20(3):261-267.

21. Othman SS, Abdul Kadir K, Hassan J, Hong GK, Singh BB, Raman N. High prevalence of thyroid function test abnormalities in chronic schizophrenia. Aust N Z J Psychiatry. 1994;28(4):620-624.

22. Shima S, Yano K, Sugiura M, Tokunaga Y. Anticerebral antibodies in functional psychoses. Biol Psychiatry. 1991;29(4):322-328.

23. Heath RG, McCarron KL, O'Neil CE. Antiseptal brain antibody in IgG of schizophrenic patients. Biol Psychiatry. 1989;25(6):725-733.

24. Henneberg AE, Horter $S$, Ruffert $S$. Increased prevalence of antibrain antibodies in the sera from schizophrenic patients. Schizophr Res. 1994; 14(1):15-22.

25. Borda T, Gomez R, Berría MI, Sterin-Borda L. Antibodies against astrocyte M1 and M2 muscarinic cholinoceptor from schizophrenic patients' sera. Glia. 2004;45(2):144-154.

26. Tanabe M, Kanehisa M. Using the KEGG database resource. Curr Protoc Bioinformatics. 2012; Chapter 1:Unit 1.12. 
27. Kamburov A, Stelzl U, Lehrach H, Herwig R. The ConsensusPathDB interaction database: 2013 update. Nucleic Acids Res. 2013;41(Database issue):D793-D800.

28. Jupe S, Akkerman JW, Soranzo N, Ouwehand WH. Reactome - a curated knowledgebase of biological pathways: megakaryocytes and platelets. J Thromb Haemost. 2012;10(11):2399-2402.

29. Schaefer CF, Anthony K, Krupa S, Buchoff J, Day M, Hannay T, Buetow KH. PID: the Pathway Interaction Database. Nucleic Acids Res. 2009;37(Database issue):D674-D679.

30. Kamburov A, Pentchev K, Galicka H, Wierling C, Lehrach H, Herwig R. ConsensusPathDB: toward a more complete picture of cell biology. Nucleic Acids Res. 2011;39(Database issue):D712-D717.

31. Allen NC, Bagade S, McQueen MB, et al. Systematic meta-analyses and field synopsis of genetic association studies in schizophrenia: the SzGene database. Nat Genet. 2008;40(7):827-834.

32. Barrett T, Wilhite SE, Ledoux P, et al. NCBI GEO: archive for functional genomics data sets-update. Nucleic Acids Res. 2013;41(Database issue):D991-D995.

33. Subramanian A, Kuehn H, Gould J, Tamayo P, Mesirov JP. GSEA-P: a desktop application for Gene Set Enrichment Analysis. Bioinformatics. 2007;23(23):3251-3253.

34. Kim K, Jutooru I, Chadalapaka G, et al. HOTAIR is a negative prognostic factor and exhibits pro-oncogenic activity in pancreatic cancer. Oncogene. 2013;32(13):1616-1625.

35. Gene Set Enrichment Analysis [homepage on the Internet]. GSEA User Guide [updated 2012 Dec. cited 2018 Nov 26]. Available from: http:// software.broadinstitute.org/gsea/doc/GSEAUserGuideFrame.html. Accessed November 26, 2018.

36. Cappello F, de Macario EC, Zummo G, Macario AJ. Immunohistochemistry of human Hsp60 in health and disease: from autoimmunity to cancer. Methods Mol Biol. 2011;787:245-254.

37. Gupta S, Mukherjee S, Syed P, et al. Evaluation of autoantibody signatures in meningioma patients using human proteome arrays. Oncotarget. 2017;8(35):58443-58456.

38. Escribese MM, Barber D. New insight into cancer immunotherapy. Allergol Immunopathol (Madr). 2017;45(Suppl 1):50-55.

39. Tosoni A, Franceschi E, Pasquini E, et al. Immunotherapy in head and neck cancer: evidence and perspectives. Immunotherapy. 2017;9(16): $1351-1358$

40. Rudqvist NP, Pilones KA, Lhuillier C, et al. Radiotherapy and CTLA-4 blockade shape the TCR repertoire of tumor-infiltrating T cells. Cancer Immunol Res. 2018;6(2):139-150.

41. Domblides C, Antoine M, Hamard C, et al. Non-small cell lung cancer from HIV-infected patients expressed PD-L1 with marked inflammatory infiltrates. AIDS. 2017;32(4):461-469.

42. Reale M, Patruno A, De Lutiis MA, et al. Dysregulation of chemocytokine production in schizophrenic patients versus healthy controls. BMC Neurosci. 2011;12(1):13.

43. Levite M, Chowers Y. Nerve-driven immunity: neuropeptides regulate cytokine secretion of $\mathrm{T}$ cells and intestinal epithelial cells in a direct, powerful and contextual manner. Ann Oncol. 2001;12 (Suppl 2): S19-S25.

44. Elenkov IJ, Wilder RL, Chrousos GP, Vizi ES. The sympathetic nervean integrative interface between two supersystems: the brain and the immune system. Pharmacol Rev. 2000;52(4):595-638.

45. Cui GB, An JZ, Zhang N, Zhao MG, Liu SB, Yi J. Elevated interleukin-8 enhances prefrontal synaptic transmission in mice with persistent inflammatory pain. Mol Pain. 2012;8:11.

46. Lee KH, Kronbichler A, Park DD, et al. Neutrophil extracellular traps (NETs) in autoimmune diseases: a comprehensive review. Autoimmun Rev. 2017;16(11):1160-1173.

47. Soliman E, Henderson KL, Danell AS, Van Dross R. Arachidonoylethanolamide activates endoplasmic reticulum stress-apoptosis in tumorigenic keratinocytes: role of cyclooxygenase-2 and novel J-series prostamides. Mol Carcinog. 2016;55(2):117-130.
48. Yang $\mathrm{X}, \mathrm{Xu} \mathrm{Y}$, Wang $\mathrm{T}$, et al. Inhibition of cancer migration and invasion by knocking down delta-5-desaturase in COX-2 overexpressed cancer cells. Redox Biol. 2017;11:653-662.

49. Yan D, An G, Kuo MT. C-Jun N-terminal kinase signalling pathway in response to cisplatin. J Cell Mol Med. 2016;20(11):2013-2019.

50. Koch P, Gehringer M, Laufer SA. Inhibitors of c-Jun N-terminal kinases: an update. J Med Chem. 2015;58(1):72-95.

51. Zhao HF, Wang J, Tony To SS. The phosphatidylinositol 3-kinase/Akt and c-Jun N-terminal kinase signaling in cancer: alliance or contradiction? (Review). Int J Oncol. 2015;47(2):429-436.

52. Wang L, Zhang C, Xu J, Wu H, Peng J, Cai S, He Y. CXCL1 gene silencing inhibits HGC803 cell migration and invasion and acts as an independent prognostic factor for poor survival in gastric cancer. Mol Med Rep. 2016;14(5):4673-4679.

53. Fond G, Macgregor A, Attal J, Larue A, Brittner M, Ducasse D, Capdevielle D. Antipsychotic drugs: pro-cancer or anti-cancer? A systematic review. Med Hypotheses. 2012;79(1):38-42.

54. Jablensky A, Lawrence D. Schizophrenia and cancer: is there a need to invoke a protective gene. Arch Gen Psychiatry. 2001;58(6):579-580.

55. Barak Y, Achiron A, Mandel M, Mirecki I, Aizenberg D. Reduced cancer incidence among patients with schizophrenia. Cancer. 2005; 104(12):2817-2821.

56. Mortensen PB. The incidence of cancer in schizophrenic patients. J Epidemiol Community Health. 1989;43(1):43-47.

57. Lin GM, Chen YJ, Kuo DJ, et al. Cancer incidence in patients with schizophrenia or bipolar disorder: a nationwide population-based study in Taiwan, 1997-2009. Schizophr Bull. 2013;39(2):407-416.

58. Markowska A, Lubin J, Markowska J, Kasprzak B, ChajewskaCzekańska M, Madry R, Stawicka M. Multiple primary cancers in BRCA $1 / 2$ carriers - a review of literature and our observations. Eur J Gynaecol Oncol. 2017;38(3):361-363.

59. Weir HK, Johnson CJ, Ward KC, Coleman MP. The effect of multiple primary rules on cancer incidence rates and trends. Cancer Causes Control. 2016;27(3):377-390.

60. Kong P, Wu R, Lan Y, et al. Association between mismatch-repair genetic variation and the risk of multiple primary cancers: a metaanalysis. J Cancer. 2017;8(16):3296-3308.

61. Brown JS. Cancer immune equilibrium and schizophrenia have similar interferon- $\gamma$, tumor necrosis factor- $\alpha$, and interleukin expression: a tumor model of schizophrenia. Schizophr Bull. 2016;42(6):1407-1417.

62. Liang F, Zhang S, Xue H, Chen Q. Risk of second primary cancers in cancer patients treated with cisplatin: a systematic review and metaanalysis of randomized studies. BMC Cancer. 2017;17(1):871.

63. Lim MC, Won YJ, Lim J, et al. Second primary cancer after diagnosis and treatment of cervical cancer. Cancer Res Treat. 2016;48(2):641-649.

64. Youlden DR, Youl PH, Peter Soyer H, Fritschi L, Baade PD. Multiple primary cancers associated with Merkel cell carcinoma in Queensland, Australia, 1982-2011. J Invest Dermatol. 2014;134(12):2883-2889.

65. Hasan A, Falkai P, Wobrock T, et al; WFSBP Task Force on Treatment Guidelines for Schizophrenia. World Federation of Societies of Biological Psychiatry (WFSBP) guidelines for biological treatment of schizophrenia - a short version for primary care. Int J Psychiatry Clin Pract. 2017;21(2):82-90.

66. Hodgson R, Wildgust HJ, Bushe CJ. Cancer and schizophrenia: is there a paradox? J Psychopharmacol. 2010;24(4 Suppl):51-60.

67. Ji J, Sundquist K, Ning Y, Kendler KS, Sundquist J, Chen X. Incidence of cancer in patients with schizophrenia and their first-degree relatives: a population-based study in Sweden. Schizophr Bull. 2013;39(3): $527-536$. 
Neuropsychiatric Disease and Treatment

Dovepress

\section{Publish your work in this journal}

Neuropsychiatric Disease and Treatment is an international, peerreviewed journal of clinical therapeutics and pharmacology focusing on concise rapid reporting of clinical or pre-clinical studies on a range of neuropsychiatric and neurological disorders. This journa is indexed on PubMed Central, the 'PsycINFO' database and CAS,

and is the official journal of The International Neuropsychiatric Association (INA). The manuscript management system is completely online and includes a very quick and fair peer-review system, which is all easy to use. Visit http://www.dovepress.com/testimonials.php to read real quotes from published authors.

Submit your manuscript here: http://www.dovepress.com/neuropsychiatric-disease-and-treatment-journal 